been recorded. D'Astros, in the section of his monograph which deals with renous obstruction, does not mention the formation of such clots. Browning $\dagger$ has reported the discovery of an organised thrombus, $\frac{1}{3}$ in. long, lying in the straight sinus, with another in the longitudinal sinus (? superior or inferior) in a less acivanced condition. His patient was a girl aged 6 , who died three months after hydrocephalus was first observed.

Hither Green, S.E.

\section{THE ACTION OF SCHARLACH R UPON X RAYED SKIN.}

\section{By J. O. WAKELIN BARRATT, M.D., D.Sc. LoNd. \\ (From the Cancer Research Laboratory, The University, Liverpool._Mrs. Sutton Timmis Memorial.)}

IN previous communications the condition of mitosis in epithelium caused to proliferate by the action of Scharlach $\mathbf{R}^{1}$ has been studied, and also the course of events following upon implantation of such epithelium. ${ }^{2}$ The present paper deals with the effect of injection of Scharlach $R$ into the skin of the ear of the rabbit exposed, 2 to 15 months previously, to the action of $X$ rays. In such cases the full destructive effect of the $X$ rays has been produced and the skin has again passed into a stationary condition.

It will be of advantage to study first the change produced by Scharlach $R$ in the $X$ rayed skin of the ear when the amount of destruction effected by the action of $\mathrm{X}$ rays has not been sufficient to produce complete disappearance of all the appendages of the skin. These changes are illustrated in

FIG. 1.
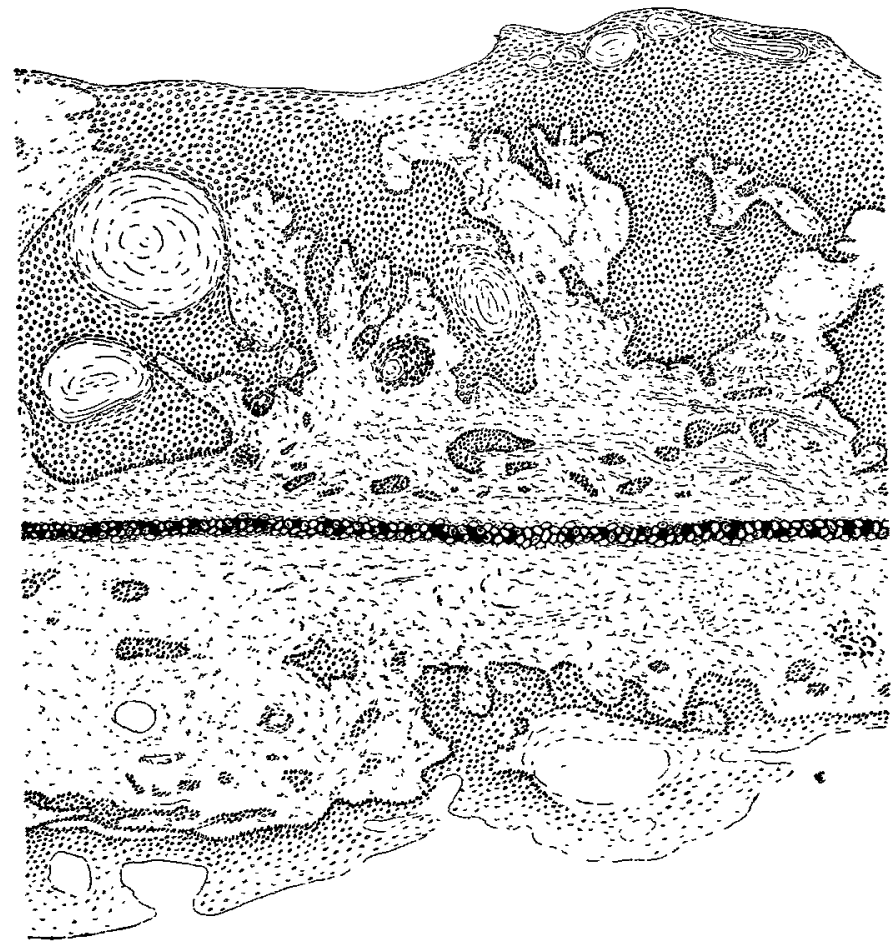

$$
{ }_{0 m i m}
$$

(The scale of Figs. 2 and 5 is the same as that of Fig. 1.)

Seation of ear of rabbit $\mathrm{A}$, exposed to the action of $\mathrm{X}$ rays 15 months previously. Scharlach $\mathbf{R}$ dissolved in olive oil was injected beneath the skin of the outer surface of the ear and later a portion of the injected area was removed for sectioning. Considerable epithelial proliferation has occurred chiefly, but not exclusively, in the epithelium of the outer surface of the ear. Both surfaces are irregular. The nuclei of the cutis vera are increased in number. The upper border of the section represents the outer The upper $\times 30$.

* D'Astros : Les Hydrocéphalies, Paris, 1898

$\dagger$ Browning: New York Journal of Nervous and Mental Disease, 1887. vol. Xiv., p. 260

1. J. O. Wakelin Barratt: On Mitoses in Proliferating Epithelium, Proceedings of the Royal Society, 1907, Series B, vol. Ixxix., p. 372

2 J. O. Wakelin Barratt: Implantation of Actively Proliferating Epithelium, ibick., p. 546 .
Figs. 1 to $4,{ }^{3}$ which are made from two rabbits (A and $B$ ) similarly treated. The changes induced by the action of $\mathrm{X}$ rays in these two rabbits were of long standing, having: been set up 15 months previously, so that at the time of injection of Scharlach $R$ about 80 per cent. of the hairs had disappeared from the portion of the ear $\mathrm{X}$ rayed, those remaining being free from pigment, and shorter and finer than those present before the application of $\mathrm{X}$ rays. The skin of the ear was thinner than normal, pale and smooth, presenting a somewhat scarred aspect and exhibiting irregular areas of increased pigmentation alternating with areas nearly free from pigment. These changes were more marked on the outer aspect of the ear than on the inner.

On microscopic examination of the $X$ raved skin before the injection of Scharlach R (Figs. 2 and 4) it was found that a marked degree of destruction of the hair follicles had occurred. This was greatest in rabbit $\mathbf{A}$ (Fig. 2). 'The hair follicles remaining were diminished in diameter and in length, the former change affecting chiefly the deeper

FIG. 2.

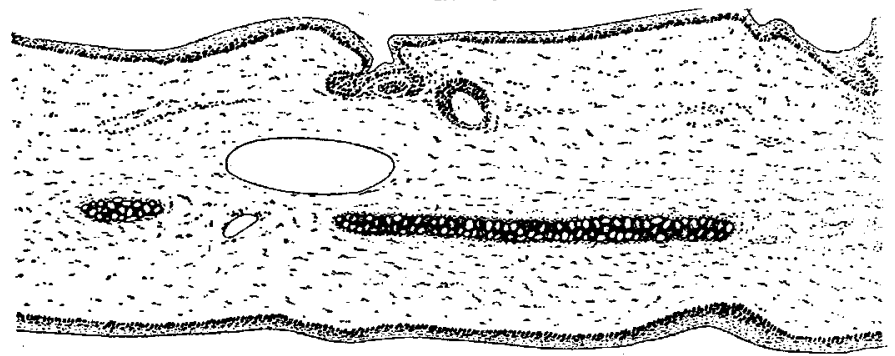

Section of ear of rabbit $A$, exposed to the action of $X$ rays 15 months previously. The portion of the ear represented months previously. The portion of the ear represented. made but is outside the area injected with Scharlach R The hair follicles have been, for the most part, destroyed by the action of $X$ rays. The surface epithelium is markedly pigmented, especially on the outer aspect of the ear. A large vein is seen above the cartilage, which does not extend completely through the section. This section should be
compared with that given in Fig. $1 . \times 30$.

portion of the follicles, which were not unfrequently converted into thin cord-like structures. Most of the papillæ of the hair follicles had disappeared, and, as a rule, hair shafts were absent. In the upper part of the follicles a lumen was present containing epithelial scales, the accumulation of which sometimes caused distension of this part of the follicle. In the lower part of the follicle, in most cases, no lumen was recognisable and the follicle appeared to be solid, being made up of small prickle cells. The mouths of the hair follicles were observable as large openings, usually filled with loose horny epithelium. Sometimes the horny epidermis at, or just below, the mouths of the hair follicles had accumulated to such an extent as to give rise to epithelial collections reaching a millimetre or more in diameter. The sebaceous glands had in part disappeared. The hair follicles were more affected on the outer surface of the ear than on the inner. The surface epithelium shown in Fig. 2 is of about the normal thickness, but is pigmented to an excessive degree. The cutis vera, owing to the small size and number of hair follicles, is thinner than normal, and appears to be made up to a greater extent than usual of connective tissue. The blood-vessels are less numerous than usual; in consequence of this the cellular elements of the corium are fewer than usual.

In rabbit $\mathrm{B}$ (Fig. 4) the destruction of the hair follicles is less marked. It is little evident in the skin of the inner surface of the ear (lying below in the figure) in which the hair follicles mostly contain hair shafts of fair size, but in the outer skin of the ear the hair follicles are affected to a greater degree, fewer hair shafts being seen. The epithelial distension of the mouths of the hair follicles referred to above is exhibited in a marked degree. The surface epithelium differs from that in Fig. 2 in not being pigmented to an excessive degree. The number of cells in the corium is not diminished to the same extent as that in the previous animal.

The injection of Scharlach $R$ into the skin of the rabbit's ear, exposed, as above described, to the action of $\mathrm{X}$ rays in.

3 The tissues from which the sections were made were hardened in Zenker's solution and stained by Heidenhain's iron-alum hæmatoxrlin method or with thionin and orange $G$. 
amount sufficient to cause partial destruction of the hair follicles, is followed by an extreme degree of epithelial proliferation such as occurs in the normal epithelium of the rabbit's ear similarly injected. In such cases the ear gradually increases in thickness at the site of injection, proliferation of the horny layer takes place, arlherent white scales appearing on the surface. At the end of 10 to 14 days the skin of the ear may present a warty character, resembling that often exhibited by surface epithelium. When the horny epidermal accumulations are removed a moist, irregular surface is exposed; complete removal of the epidermic scales is, however, difficult, for plugs may pass downwards into the proliferated prickle layer, portions of which may be torn off in the course of attempted removal of the horny epidermal accumulations.

On microscopic examination of the section obtained from rabbit A (Fig. 1) it is seen that an extreme degree of proliferation of the rete Malpighii and of the prickle layer of the hair follicles has taken place, the proliferation being of two kinds: (1) a simple more or less regular increase in the

FIG. 3.

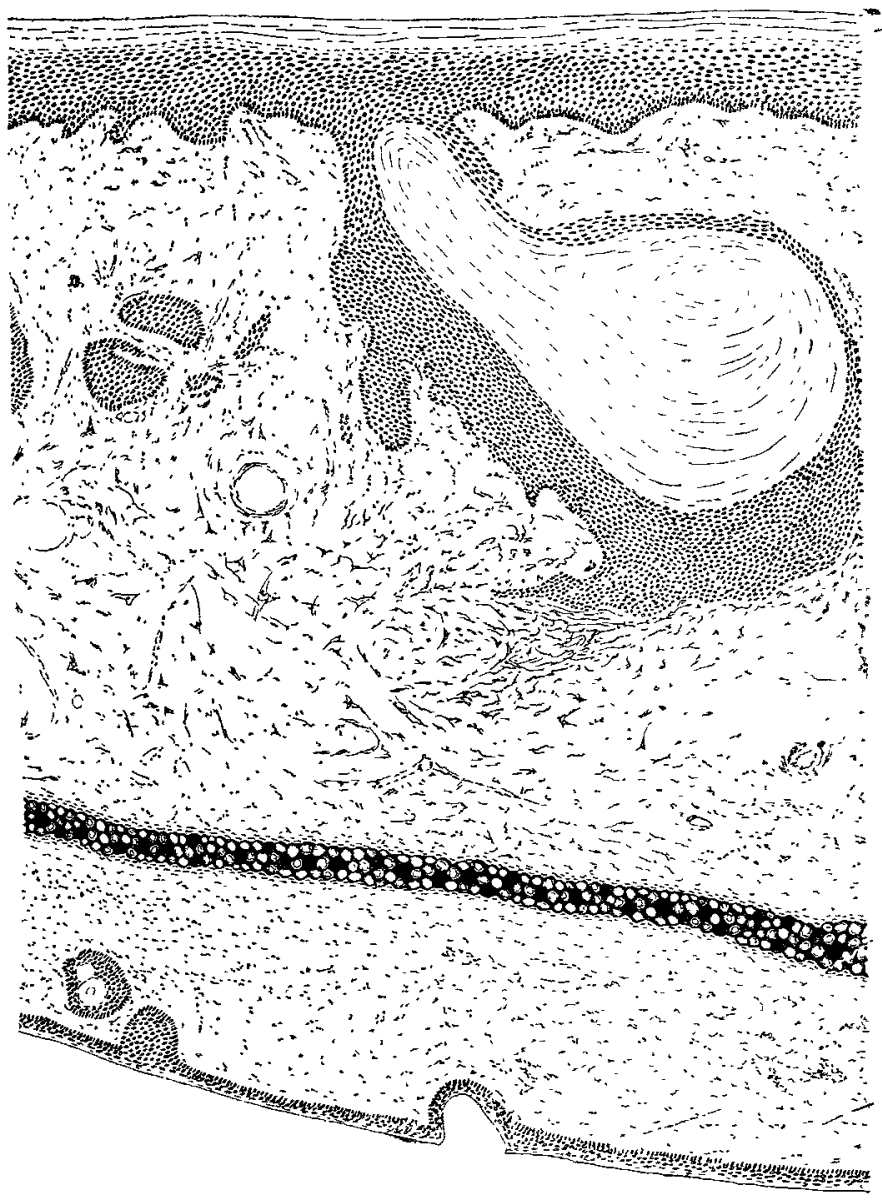

Section of the ear of rabbit $B$, exposed to the action of $X$ rays 15 months previously. Scharlach $R$ was injected beneath 15 months pheveath the skin of the outer surface of the ear, and 14 days later a portion of the injected area was removed for sectioning. Marked epithelial proliferation and an increased production of connective tissue are seen in the upper part of the In the lower part no marked change is observable. $\times 30$.

number of epithelial cells ; and (2) an irregular downgrowth or outgrowth of epithelium, produced under the influence of adjoining globules of oil containing Scharlach $\mathbf{R}$ in solution and strikingly resembling that seen in epithelioma. The epithelial proliferation is marked on both sides of the central cartilage of the ear, but is more marked in the skin of the outer surface of the ear (upper surface in the figure) than in that covering the inner surface. The proliferation of the epithelial covering of the inner surface of the ear was caused by some of the solution of Scharlach $R^{4}$ injected into the connective tissue lying upon the outer surface of the cartilage passing into the connective tissue covering the inner surface of the cartilage. (In rabbit $B$ it will be seen that this did not occur.) The enlargement of the lumen of the mouths of the hair follicles is obviously due to the very large general increase of the prickle epithelium and not to pressure from within due to an accumulation of horny scales, although such accumulation does occur to some extent. No hair shafts are seen in the section.

In the section obtained from rabbit $B$ (Fig. 3) the epidermal proliferation is confined to the skin covering the outer surface of the ear, the whole of the Scharlach $\mathbf{R}$ injected lying external to the cartilage of the ear, whereby its action was prevented from extending to the inner surface (lower surface in the figure). In this section marked increase of the epithelium of the surface and of the mouths of the

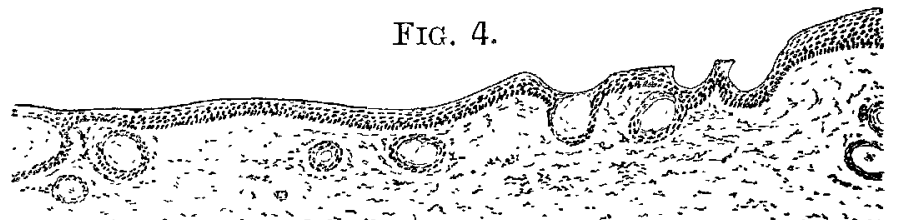

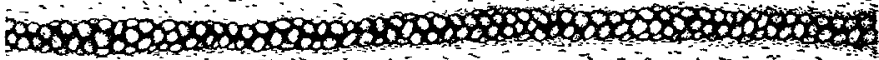

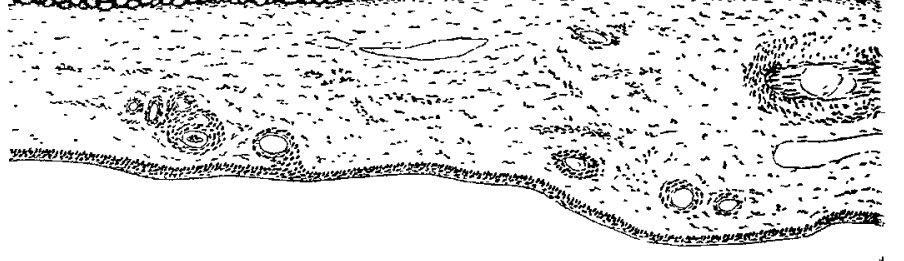

Section of ear of rabbit $B$ exposed to the action of $X$ rays 15 mont hs previously. The portion of the ear represented lies close to that from which the previous section has been made, but is outside the area injected with Scharlach $R$. The hair follicles are destroyed to a less extent than is the case in the section represented in Fig. 2. The clestruction is greater in the outer skin of the ear (lying above the cartilage) than in the inner. This section should be compared with that given in Fig. $3 . \times 30$.

hair follicles is observable. One of the latter has undergone very considerable enlargement, the mode of origin of which will be understood on reference to Fig. 4, taken from the adjoining part of the ear free from Scharlach $\mathrm{R}$, which may therefore be taken to represent the condition of the ear before injection. In the latter figure it will be observed that the empty months of the hair follicles are, as already mentioned, of large size, though the follicles themselves have become in part atrophied or destroyed. The enlargement of the former, when it occurs, is therefore all the more striking. In this section, as in that shown in Fig. 1, a few sebaceous glands of small size can be recognised. It should be observed that injection of Scharlach $R$ does not cause the appearance of proliferation in the cells of the sebaceous glands.

If Scharlach $R$ is injected into the ear of a rabbit which has many months previously been subjected to severe

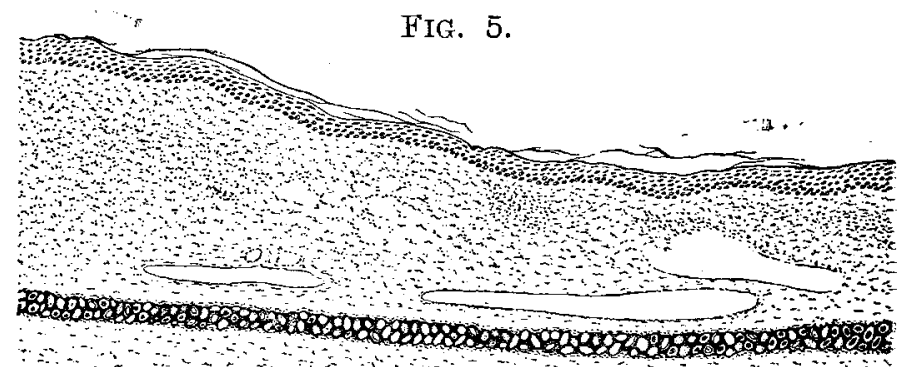

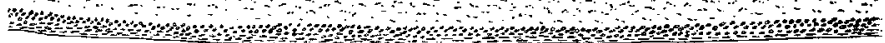

Section of ear of rabbit subjected to serere $\mathrm{X}$ raying 67 clays previously. Ten days before the portion of tissue, from which the section is made, was excised a small amount of Scharlach $R$ was injected into the subcutaneous tissue between the outer skin and the cartilage. A moderate degree of epithelial proliferation has occurred as the result of the injection. Note that the hair follicles and sebaceous glands have been completely destroyed. The cutis vera is irregularly and tend to form localised collections. $\times 30$.

$\mathrm{X}$ raying, then a result is obtained which differs in some respects from that above described. If the skin of the ear has been sufficiently $X$ rayed, all the appendages of the skin are destroyed, and the corium, which is sometimes thinner than normal, sometimes of somewhat increased thickness, containing a fair number of nuclei, is found to be made up 
of fibrous tissue covered externally by a uniform layer of epithelium, generally thicker than that observed in the normal condition (that is to say, $40 \mu$ to $50 \mu$ thick, the usual thickness being about $25 \mu$ ). The condition of such $\mathrm{X}$ rayed skin is comparable to that of deeply scarred skin.

After the injection of a small amount of Scharlach $R$ into such $X$ rayed skin proliferation of the surface epithelial covering is observed to the extent represented in Fig. 5 . In its character this epithelial increase is similar to the surface

FIG. 7 .
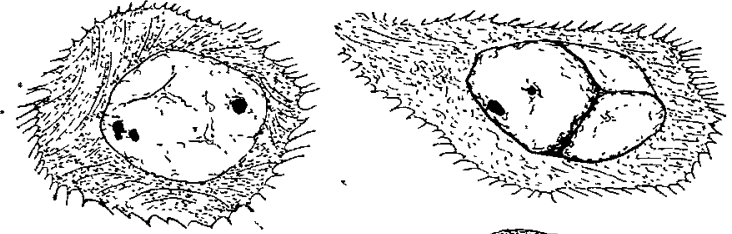

FIG. 8.

FIG. 6 .
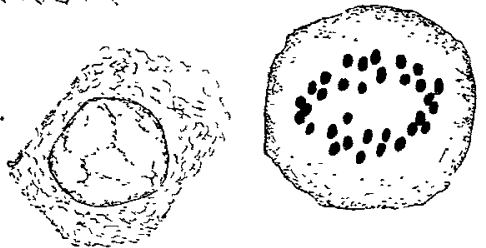

FIG. 9.

Fra. 6.-Normal prickle cell from Fig. 2. For comparison with the cell shown in the succeeding figure. $\times 1000$.

FIG. 7.-Prickle cell from proliferating epithelium shown in Fig. 1 . The cell generally is enlarged, both nucleus and cytoplasm being of more than the normal dimensions. The prickles, which are prominent, are thinned and drawn out, the prickle spaces being correspondingly increased in size. In the cytoplasm some indications of the epidermal fibres are recognisable. $\times 1000$

FIG. 8.-Prickle cell from the proliferated epithelium shown in Fig. 1, exhibiting fragmentation of the nucleus into three parts. $\times 1000$.

FIG. 9.-Mitotic figure from the proliferated epithelium shown in Fig. 1. The chromosomes, more or less oral in form, are about 30 in number, and are arranged upon the equatorial plane of a spindle (not shown in the figure). $\times 1000$.

increase seen in Figs. 1 and 3. It does not, however, progress much further than is shown in Fig. 5, owing to the fact that the effect of the injection of more than a relatively small amount of Scharlach $R$ is to cause necrosis. The fact brought out by the injection in such cases is, that Scharlach $\mathbf{R}$, as would be expected from the results previously obtained, is still effective when its action is exerted exclusively upon skin exhibiting only surface epithelium, the appendages of the skin having completely disappeared.

The degree of $\mathrm{X}$ raying just described represents the limit available for experimental work, the stage beyond being that

Fig. 10.

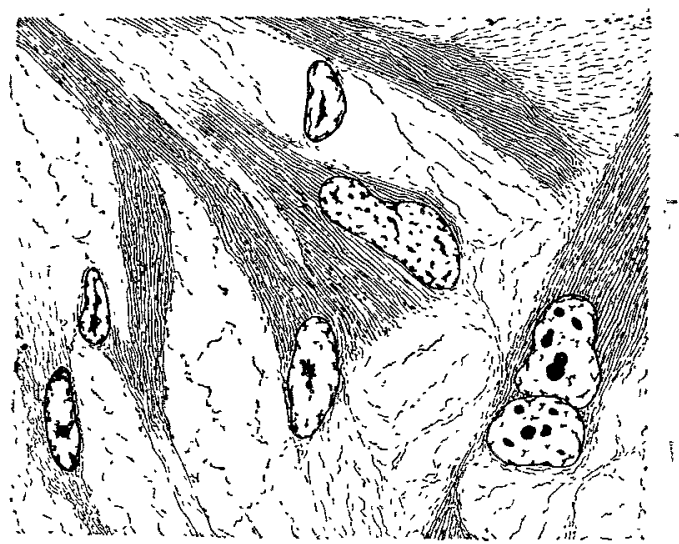

Portion of cutis vera of Fig. 3 more highly magnified Lying upon and among the connective tissue bundles (unstained) are numerous flattened cells, the cytoplasm of which is thin, lightly stained, and spread out upon the connective
tissue fibres. $\times 650$.

in which the surface epithelium is broken and sloughing may resuit. It is, however, difficult to carry out experimental work upon skin of the type shown in Fig. 5, even when the immediate effect of $X$ raying has long passed off, for such skin remains in a condition of lowered vitality and is readily affected by injurious influences.

A marked feature of the action of Scharlach $\mathbf{R}$ upon the skin of the ear of the rabbit, subjected many months previously to the action of $X$ rays, is its tendency to cause necrosis, even though the degree of $X$ ray injury may not exceed that shown in Figs. 2 and 4 . Blistering of the surface is readily produced, a crust forms, and the area of skin injected may pass slowly into a condition of dry gangrene. Should this occur a line of separation appears at the edges of the injected area, and ultimately the necrotic portion is shed, the ear showing a large hole when healing is complete. Suppuration does not occur, nor is any acute inflammatory process observable. The animal does not scratch the ear, and seems quite unconscious of the occurrence of any morbid process.

The tendency to sloughing is obviously related to the degree of destruction of the epithelial appendages of the ear. Eren with slight degrees of $\mathbf{X}$ rays. the injection of large amounts of Scharlach $\mathbf{R}$ is likely to be followed by vesiculation, while in old $X$ rayed ears in which severe changes have been produced it is difficult to avoid vesiculation, even though only a small amount of Scharlach $R$ is injected. In consequence the opportunities of studying changes in the latter case are somewhat limited.

The characters of the changes in the proliferated epithelial cells following upon the injection of Scharlach $R$ in old $X$ rayed skin of the rabbit's ear are also similar to those following injection into normal skin. The proliferating cells of the prickle epidermis and of the hair follicles are increased in size, the increase affecting all parts of the cells. The nuclei generally measure about $12 \mu$ instead of about $9 \mu$ in normal prickle cells. (Figs. 6 and 7.) Fragmentation of the nuclei into two or more parts, not howerer always

FIG. 11.

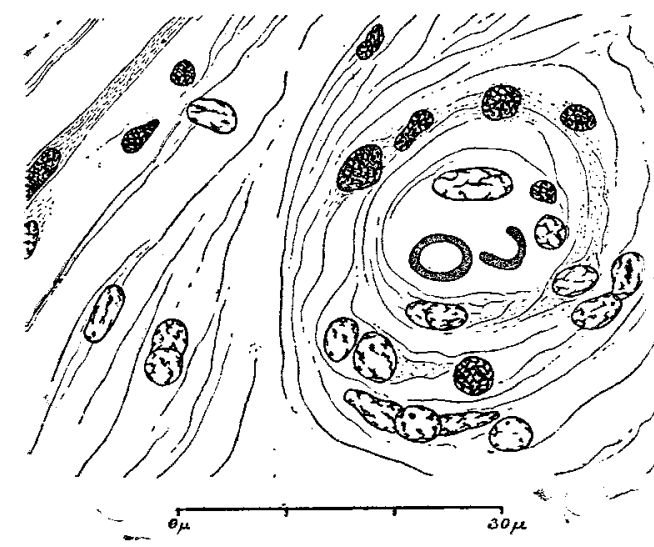

Portion of cutis vera of Fig. 3 more highly magnified, To the right a blood capillary is seen containing two red blood cells, in optical section. In addition to the endothelial cells, in optical section. In addition to the endothelia lightly stained, several smaller darkly stained free nucle lightly stained, several smaller darkly stained free nucle are seen, chiefiy disposed round the capillary ressel. The larger elastic fibres is partly indicated. $\times 500$.

completely separated, is not uncommon. (Fig. 8.) The prickles are unusually distinct and the prickle spaces large. Indications of the epidermal fibres are more or less obtrusive. (Fig. 7.) Mitosis, which is fairly common, is characterised by the appearance of short oval chromosomes; these resemble those in the reduced division of epithelial cells already described. ${ }^{5}$ In Fig. 9, which illustrates their appearance, about 30 chromosomes are present. I have not observed rod-like chromosomes in the sections made In many of the mitoses the chromosomes were not sufficiently separated from one another to enable the type to be identified. The cells of the sebaceous glands disappeared after the injection of Scharlach $R$. No pigmented cells were visible, after injection, in the proliferated epithelium of rabbit $A$, though prior to injection of Scharlach $R$ these were present in considerable numbers. (Compare Figs. 1 and 2.)

In the cutis vera, after the injection of Scharlach $R$ into old $X$ rayed skin, the connective tissue was found to be increased in amount (compare Figs. 1 and 2; also Figs. 3 and 4); the nuclei of the cutis vera were much more numerous than before injection. Among the latter large flat cells were obtrusive in rabbit $B$ (Figs. 3 and 10), less so in rabbit $A$. These exhibited well-defined large nuclei and darkly stained cytoplasm forming thin prolongations, often branched, lying upon and between the connective tissue

$$
\text { 5 Loc. cit., p. } 375 .
$$


fibres (Fig. 10), In addition to these, large nuclei, similar to the preceding, but not obviously flattened and not surrounded by any clearly defined cytoplasm, were common among the connective tissue fibres and in the neighbourhood of the blood-vessels (Fig. 11). Nevertheless, the number of free cells lying around the blood-vessels was much less than had been observed when the healthy skin of the rabbit's ear was injected with Scharlach $R$. Smaller nuclei, with relatively abundant chromatin, staining more darkly than the preceding (Fig. 11), are also met with in considerable numbers lying between the connective tissue bundles and also around blood-vessels; the cytoplasm of these nuclei could not be defined; multinuclear leucocytes were unfrequent. The blood-vessels of the cutis vera were diminished in number in proportion to the destructive effect of the $\mathrm{X}$ rays. Some increase in the number of bloodvessels, limited in degree, took place after injection of Scharlach $\mathbf{R}$, the increase being greater the more vascular the skin was at the time of injection. The liability of the $\mathrm{X}$ rayed skin to vesiculation and sloughing after the injection of Scharlach $R$ is presumably related to the abnormal structural character of the cutis vera. Mitoses of the somatic type are occasionally met with in the cells of connective tissue. Mast cells are sometimes encountered, but pigment cells appear to be absent.

\section{Summary.}

1. In old X rayed skin of the rabbit's ear the injection of a solution of Scharlach $\mathbf{R}$ is followed by epithelial proliferation, which may reach an extreme degree, provided the destructive effect of the $\mathrm{X}$ radiation has not been excessive.

2. A marked tendency to sloughing is observed when Scharlach $R$ is injected into old $X$ rayed tissues, the tendency being greater the more marked the extent to which the appendages of the skin have been destroyed.

Liverpool.

\section{ittedical Socretirs.}

\section{ROYAL SOCIETY OF MEDICINE.}

\section{SECTION FOR THE STUDY OF DISEASE IN CHILDREN.}

Exhibition of Clinical Cases and Pathological Specimens.

A MEETING of this section was held on Jan. 24th, Mr. A. H. TubBy, the President, being in the chair.

Dr. Anne L. J. Kann showed for Mr. Duncan C. L. Fitzwilliams a case of (?) Fibro-lipoma. It was that of a boy aged 2 years and 8 months. On examination there were four small swellings, distinct from one another, on the back of the arm and in the axilla. They felt fibrous, some being larger than others. The axilla was filled with a mass about as large as an orange, which bulged forward the anterior axillary wall and appeared in front under the pectoral muscles. It was firm, with well-defined edges and slight lobulation. It was moveable on the structures beneath and under the superficies. There was no impulse on coughing or crying. The child was very backward and undersized, with marked signs of rickets. The fontanelle was still open.

Dr. ANNE KANN also showed for Mr. Fitzwilliams a case of Lymphangioma resembling Hernia of the Lung. It was that of a boy aged 6 . The mother had noticed that something was wrong with the right side of his neck when he cried. She stated that she noticed this vaguely when she was washing the child, and she could recall it as far back as 12 months ago, but she took little notice of it until it began to get larger during the last three months. In November the child got whooping-cough, and this lasted until the end of December. During this time his mother noticed that the lump got very large in his neck during the spasms of coughing. On examination there was a fulness of the root of the neck above the right clavicle and behind the sterno-mastoid muscle. It was ill-defined and quite soft, and small nodules could be felt in it. It seemed to disappear under the fingers towards the region of the subclavian artery. On coughing or straining the root of the neck filled up and bulgéd in a prominent manner, and on palpation the tumour again subsided. Though breath sounds were heard they were probably transmitted. The condition at first sight was extremely like a hernia of the apex of the right lung, but there was no indrawing on deep inspiration, nor could any ring be felt in the deep part of the neck. The diagnosis was that the condition was an extensive lymphangioma with large loculi, the greater portion of the tumour being situated in the chest.

Dr. R. C. JewesbuRY showed cases of two similar but somewhat unusual heart conditions in sisters. 1. Aged eight. The mother was told by her doctor that this child's "heart was affected" when she was three weeks old. The child was not cyanosed at birth and had never been noticed to get blue. She had suffered from dyspncea on exertion for the last three to four years, and had complained of growing-pains during the last two years. There was no history of acute rheumatism. The heart was enlarged, its apex beat being in the sixth space in the nipple line. There was a systolic murmur at the apex conducted out. The second sound was accentuated. In the second and third left space a loud, zumbling murmur, partly presystolic and partly systolic, was heard, and a well-marked thrill was felt in this position. At the pulmonary area a systolic murmur was present, conducted upwards to the neck. Aortic sounds : first sound soft, otherwise normal. 2. Aged 10 years. No heart disease was ever suspected until she was brought to hospital a few days before. She had always been a healthy child. There was no history of rheumatism; the heart was not enlarged. In the second and third spaces a well-marked systolic thrill was felt, and over this area a prolonged, continuous, booming murmur was present, very similar to that heard in her sister's case.

Mr. R. C. ELMSLIE showed three cases of an unusual form of Disease of the Hip-joint- "Calvé's Psendocoxalgie." 1. A boy, aged 10. In November, 1909, he fell and twisted his right foot, and attended an out-patient department for a week and recovered. About three weeks later he was kicked or the right hip, which was noticed by the mother to be much bruised ; from that time he limped and had occasional pain in the knee, especially after lying down. He had no pain at night. In the following March he came to the hospital. At that time the hip movements were free, except full hyperextension; the limb was 3 in. shorter than the left, the shortening being above the great trochanter; there was little wasting of the thigh. A skiagram showed that the acetabulum was clear and smooth, but that the epiphysis of the head of the femur was a little flattened, its surface uneven, and its upper edge proportionately thicker than it should be; the neck of the femur appeared shorter and thicker than the normal. He was kept in bed for three weeks, and the condition did not alter. He was then allowed up. A fresh skiagram taken in May showed no alteration. After this he was kept under occasional observation until February, 1912, during which time the condition did not alter appreciably. Subsequent skiagrams showed no important change, although the flattening of the epiphysis and the thickening of its upper border became rather more evident. 2. A boy aged 8. The parents in this case had not noticed anything wrong, but were informed after a school medical inspection that the boy limped. He was brought to the hospital in January, 1912. At that time there was a little limitation of the abduction of the right hip-joint, most marked in the flexed position; other movements were free. The shortening was very slight, not more than $\frac{1}{4}$ in., and there was a little wasting in the thigh. A skiagram was taken, and the appearances were found practically identical with those in the last case. The parents were then informed that no treatment would be necessary. These two cases agreed as to clinical signs and skiagraphic appearances with Calve's description, but neither was observed in the acute stage described by that author. The third case presented, however, some differences. 3. A girl aged 9. At the beginning of 1912 this chila had an attack of St. Vitus's dance and rheumatism in the right leg. In the summer she was an in-patient for St. Vitus's dance at a hospital. She was transferred to a fever hospital for diphtheria in August. While convalescent of the attack of diphtheria she was noticed for the first time to limp, but she had at this time no pain or disability. When she returned from a convalescent home the mother noticed the $\operatorname{limp}$, and brought her to St. Bartholomew's Hospital in December. No injury to the hip could be remembered. The right hip was kept flexed, further flexion was free, extension 